Artículo de revisión

\title{
Atención y conciencia visual
}

\author{
Attention and visual consciousness \\ Francisco Pereira Gandarillas ${ }^{1^{*}}$
}

1 Departamento de Filosofía, Universidad Alberto Hurtado. Santiago, Chile.

\section{Resumen}

Algunos filósofos y científicos cognitivos piensan que la atención es necesaria para la conciencia visual. Ellos normalmente aceptan este requerimiento sobre la base de estudios sistemáticos de patologías perceptuales, como el síndrome de negligencia unilateral, o respaldados en experimentos psicológicos de ceguera inatencional en los que individuos con sus capacidades perceptivas intactas supuestamente no experimentan conscientemente elementos presentes en su campo visual cuando estos son desatendidos. En este artículo sugeriré que la estrategia de la ceguera inatencional es insuficiente y no nos fuerza a aceptar que la atención es necesaria para la conciencia visual por dos razones. Primero, la evidencia empírica disponible es consistente con interpretaciones alternativas que no apelan a la necesidad atencional. Segundo, hay buenas razones para pensar que la atención es independiente de la conciencia. Si esto es así, entonces posiblemente existen modos de conciencia que no requieren de atención.

Palabras clave: atención, conciencia visual, ceguera inatencional

\begin{abstract}
Some philosophers and cognitive scientists think that attention is necessary for visual consciousness. They normally accept this requirement on the basis of systematic studies of perceptual pathologies, such as unilateral neglect, or based on psychological experiments of intentional blindness in which individuals with intact perceptual capacities allegedly don't experiment at the conscious level features which are present in their visual fields but not attended. In this paper I will suggest that the inattentional blindness strategy is insufficient and do not force us to accept that attention is necessary for visual consciousness for two reasons. First, the empirical evidence available is consistent with alternative interpretations that do not appeal to the necessity of attention. Second, there are good reasons to think that attention and visual consciousness are independent. If this is so, then possibly there are modes of consciousness that do not require attention.
\end{abstract}

Keywords: attention, visual consciousness, inattentional blindness

\footnotetext{
* Correspondencia: fpereira@uahurtado.cl. Departamento de Filosofía, Universidad Alberto Hurtado, Avenida Libertador Bernardo O’Higgins 1869, 3er. piso, Santiago. Teléfono: +56 (2) 6920209

Recibido: 18-10-11. Revisión desde: 16-11-11. Aceptado: 28-12-11

DOI: $10.5839 /$ rcnp.2012.0701.05
} 


\section{Introducción}

La estrecha vinculación entre la atención y la conciencia visual se presenta hoy como un tópico de investigación fundamental para las neurociencias, la psicología cognitiva y la filosofía de la mente ${ }^{1}$. ¿Es la atención necesaria para la conciencia visual? La tesis que hasta el momento parece dominar la investigación contemporánea así lo sugiere. La atención no solo estaría vinculada o asociada a la conciencia visual, sino que sería un requisito necesario para su surgimiento.

Defensores ya clásicos del requerimiento o tesis de la necesidad atencional para el surgimiento de la conciencia (en adelante: TNA) son Mack \& Rock, quienes aseveran que "no hay percepción consciente sin atención" (1998, p. ix) y O'Regan \& Noë, que reconocen que "por definición, sin atender algo (es decir, sin estar consciente de algo) el campo visual no puede verse de modo alguno" (2001, p. 955). En los últimos años Prinz ha realizado una interpretación aún más fuerte del papel de la atención en la conciencia visual, en la cual ella sería no solo necesaria, sino también suficiente para su surgimiento:

“...la conciencia surge cuando y solo cuando atendemos. Los procesos neuronales subyacentes a la atención son los mecanismos físicos mediante los cuales los correlatos neuronales de las representaciones de nivel intermedio se hacen conscientes. ¿Cómo es que nos hacemos conscientes? Atendemos" (Prinz, 2011, p. 175).

Las motivaciones para adoptar TNA son diversas y existe una vasta literatura experimental al respecto. En general es posible identificar dos clases de consideraciones que respaldarían incluso una tesis más fuerte análoga a la sostenida por Prinz (2011). Por una parte, tenemos los estudios sistemáticos de patologías asociadas a la percepción visual, en los que destacan las investigaciones sobre el síndrome de negligencia unilateral, desorden normalmente causado por lesiones en la corteza parietal inferior derecha (Driver \& Mattingly, 1998). Los sujetos con negligencia unilateral no experimentan conscientemente su campo visual izquierdo. Por ejemplo, estos individuos no tienen conciencia de la sección izquierda de objetos macroscópicos regulares al observarlos y es frecuente que su experiencia consciente excluya secciones completas del flanco izquierdo de sus cuerpos $^{2}$. ¿Qué impide tener una experiencia consciente en el campo visual izquierdo en los casos de negligencia unilateral? Una lectura de la evidencia empírica disponible apunta a un déficit de atención. Hipotéticamente, en estos casos la concien-

\footnotetext{
1 “Atención" y "conciencia" son expresiones que están lejos de ser utilizadas de manera estándar en la literatura especializada. En efecto, la extensión de cada uno de estos términos muchas veces difiere o incluso involucra una amplia gama de situaciones cotidianas preteóricas o mecanismos de diversa índole. En ningún caso aspiro a dar una definición de estos términos en el contexto del presente artículo, pero sí me parece importante aclarar cómo los utilizaré. Hablaré de "atención" para referirme a la atención selectiva, con particular énfasis en lo que usualmente se especifica como atención selectiva endógena top-down, y usaré la expresión "conciencia" para referirme simplemente al contenido fenoménicocualitativo que caracteriza nuestras experiencias visuales.

${ }^{2}$ Es importante recalcar que esta carencia fenomenológica o de conciencia no impide que estos sujetos de hecho perciban o vean inconscientemente los objetos y propiedades localizados en su campo visual. La negligencia unilateral en ningún modo excluiría la posibilidad de actividad cortical visual inconsciente o subpersonal.
}

cia se desvanece debido a la falta de atención, incluso si la actividad perceptiva se sostiene en un dominio subpersonal ${ }^{3}$.

Una segunda clase de consideraciones que respaldarían la pertinencia de TNA se funda en experimentos psicológicos en los que un porcentaje representativo de individuos con sus capacidades perceptivas intactas no es capaz de reportar la presencia de objetos y propiedades situados en su campo visual cuando estos no son atendidos. En esta categoría se encuentra la ceguera inatencional, fenómeno que será eje fundamental del presente artículo en una de sus formulaciones experimentales más paradigmáticas: la prueba del gorila desarrollada por Simon \& Chabris (1999), bajo la condición general que ellos mismos especificaron como "opaca" ${ }^{4}$. En este experimento se solicita a un grupo de individuos observar un video de 75 segundos de duración, protagonizado por dos equipos compuestos de tres jugadores cada uno, un equipo con camiseta blanca y otro con camiseta negra. Cada uno de los equipos realiza entre sus miembros pases de mano con una pelota naranja típica de baloncesto. A los 44 segundos del montaje se produce un evento muy específico en el video: una mujer vestida con un disfraz de gorila que cubre completamente su cuerpo avanza de izquierda a derecha atravesando toda la escena en una acción que dura aproximadamente cinco segundos mientras los jugadores de cada equipo continúan jugando.

Lo interesante para nuestros propósitos es lo que sucede con los individuos que participan como observadores. Antes de comenzar el video ellos son informados que verán dos equipos jugando baloncesto y se les solicita formalmente seguir dos instrucciones precisas: (i) poner atención en el desempeño de solo uno de los equipos y (ii) contar mentalmente el número total de pases de pelota realizado por el equipo seleccionado la denominada "condición fácil" - o realizar una cuenta mental separada del número de pases con rebote y del número de pases aéreos ejecutados por el equipo atendido, tarea que constituye la denominada "condición difícil". Ciertamente, no se les informa de manera anticipada del evento del gorila.

Considerando el promedio entre la condición fácil y la condición difícil los resultados generales del experimento en su versión opaca sorprenden. Un 70,5\% de los participantes que atendieron las acciones del equipo negro se dieron cuenta y fueron capaces de reportar la presencia del gorila. La cifra es aún más llamativa en el caso de los participantes que seleccionaron el equipo blanco. Solo $46 \%$ de ellos se percataron de la presencia del gorila. Posteriormente, Simon \& Chabris (1999, p. 1069) realizaron una nueva prueba en que la aparición del gorila es aún más preponderante. Se trata de un video de 62 segundos en el cual el gorila no solo cruza el video desde el flanco izquierdo al derecho, sino que también se detiene en el centro de la escena y se golpea el pecho mirando directamente a la cámara. Pese a que la escena del gorila dura nueve segun-

\footnotetext{
${ }^{3}$ Prinz es categórico al respecto: "Se sabe que las áreas parietales inferiores del cerebro que típicamente causan este desorden juegan un rol en la implementación de la atención. La negligencia también se asocia con lesiones en los campos oculares frontales, que son estructuras de corteza frontal asociadas con la orientación de la atención (...) Los pacientes con negligencia no pueden percibir conscientemente cosas a su izquierda porque no pueden atenderlas" (2011, pp. 177178).

${ }^{4}$ La condición opaca es aquella en que los siete protagonistas del video observado por los participantes del experimento fueron filmados de manera simultánea, lo que permite en ciertos casos que se opaquen unos a otros o que opaquen la presencia de la pelota de baloncesto. Esta condición se opone a otra versión denominada "transparente" (Simon \& Chabris, 1999, p. 1066).
} 
dos, solo $50 \%$ de los participantes se dieron cuenta de su presencia en la condición fácil, resultado que es muy semejante al $42 \%$ obtenido en el experimento original bajo la misma condición fácil. Todo esto ciertamente sugiere que aproximadamente la mitad de los observadores que participan en estos experimentos no se dan cuenta de la presencia de este estímulo mientras siguen las instrucciones especificadas.

Lo sorprendente de los resultados de estos experimentos de ceguera inatencional ha llevado a investigadores como O'Regan \& Noë (2001) a adoptar TNA. Un porcentaje importante de los participantes de los experimentos no se daría cuenta y no sería capaz de reportar lingüísticamente la aparición del gorila por una razón muy simple, no serían conscientes de su presencia. En estos casos la atención estaría completamente orientada a satisfacer las demandas especificadas, es decir, seleccionar un equipo y contar el número de pases de balón que realizan sus miembros. Como los individuos no atienden el gorila, O'Regan \& Noë (2001) afirman simplemente que ellos no serían conscientes de su presencia ${ }^{5}$.

El propósito fundamental de la siguiente sección de este artículo será cuestionar las credenciales que tienen este tipo de experimentos al momento de defender una tesis fuerte de la relación entre atención y conciencia como lo es TNA. En primer lugar, mencionaré brevemente dos consideraciones generales por las cuales estos experimentos en ningún caso establecerían concluyentemente la necesidad de la atención para la conciencia visual. Por último, centraré la discusión en los casos de ceguera inatencional y argumentaré que hay antecedentes de carácter empírico y teórico que sugieren precisamente una interpretación de este fenómeno opuesta a TNA, según la cual habría formas de conciencia visual en la cual la atención no está involucrada.

\section{Desafiando TNA}

Si bien la interpretación de datos empíricos realizada por autores como Prinz (2011), O’Regan \& Noë (2001) o Mack \& Rock (1998) es sugerente, a mi juicio la evidencia discutida en ningún caso establece que la atención es siempre necesaria para la conciencia visual. Hay dos consideraciones que no debemos ignorar al evaluar los antecedentes experimentales. La primera es de orden metodológico y apunta al tipo de mediciones utilizadas en los experimentos sobre el papel de la atención en la conciencia. Las interpretaciones de los datos empíricos y los resultados de los experimentos parecen depender en gran medida de los mecanismos utilizados para detectar si hay o no conciencia y cuál es el contenido de ella. Las mediciones directas, aquellas que no requieren de ningún tipo de inferencia explicativa, como son los reportes lingüísticos y conductuales presentes en experimentos de ceguera atencional y ceguera al cambio, tienden a respaldar paradigmas de selección temprana en los cuales los observadores parecen no tener registro consciente de los estímulos no atendidos. Esto contrasta con lo que podemos observar cuando se implementan metodologías indirectas, en las que se realizan inferencias a partir de efectos específicos en los observadores, incluyendo la activación de zonas específicas en la corteza cerebral o el tiempo de reacción

\footnotetext{
${ }^{5}$ Conclusiones semejantes (Blackmore et al., 1995; Rensink et al., 2000) se han desprendido de casos de ceguera al cambio, en los cuales los sujetos no logran detectar cambios o diferencias significativas entre dos imágenes cuando estas son presentadas de manera sucesiva.
}

a estímulos. Este tipo de metodologías está a su vez estadísticamente vinculada al privilegio de paradigmas de selección tardía en los cuales los observadores sí tendrían conciencia de elementos desatendidos durante la experiencia ${ }^{6}$.

Una segunda consideración, en mi opinión aún más relevante que la anterior, es que los experimentos presentados en la sección anterior no permiten descartar interpretaciones alternativas que no implican aceptar TNA. Los casos patológicos de negligencia unilateral claramente no establecen que la atención es siempre necesaria para la conciencia visual. Para instaurar esto habría que determinar si es así en todos los casos y además descartar si en ellos son solo los mecanismos responsables de la atención los que están dañados. Prinz admite esto sugiriendo que en la negligencia unilateral siempre queda abierta la posibilidad de que las lesiones "comprometan algo diferente a la atención" (2011, p. 178) ${ }^{7}$. Por otra parte, el caso de ceguera inatencional presentado anteriormente no parece incompatible con la posibilidad de que, a pesar de no atender a la mujer disfrazada de gorila, algunos de los sujetos que participan del experimento sean conscientes de algún modo de su presencia. En efecto, investigaciones proponen que la ignorancia de la presencia del gorila es compatible con el hecho de que este "sí hace una diferencia fenoménica para el sujeto que lo desatiende" (Mole, 2009, p. 38) ${ }^{8}$.

Teniendo en cuenta las dos consideraciones anteriores, me parece fundamental introducir ahora antecedentes que tienden a respaldar positivamente una lectura opuesta a TNA, según la cual es posible que existan elementos desatendidos que sí son representados conscientemente durante una experiencia visual. Para este propósito es importante considerar el reciente trabajo experimental de Lamme (2003) y las repercusiones que este ha tenido en la filosofía de la mente y la neurociencia (Raftopoulos, 2009). Según Lamme (2003), de las diversas entradas (inputs) sensoriales que llegan a nuestro cerebro solo un grupo limitado alcanza a experimentarse conscientemente y es susceptible de ser reportado verbalmente una vez que la atención lo ha seleccionado ${ }^{9}$. Lo central en la propuesta de Lamme (2003) es que el proceso de selección atencional opera con independencia respecto de los procesos vinculados al surgimiento de la conciencia, por lo que no tiene incidencia

\footnotetext{
${ }^{6}$ Para una revisión crítica de cada una de las metodologías y su eventual integración, sugiero revisar la propuesta de Lavie (2006).

${ }^{7}$ La solución apunta a realizar experimentos -inducir los síntomas del síndrome de negligencia unilateral- en personas sin daño cerebral, mediante la manipulación e inhibición de aquellas áreas o mecanismos específicos responsables de la atención. Esta es una tarea que presenta serias dificultades, siendo quizás la más relevante la imposibilidad de detectar de manera determinada aquellas zonas o mecanismos de la corteza parietal responsables de la atención. Además, no es evidente si efectivamente hay algo así como un área o un único mecanismo responsable de la atención (Mole, 2010).

${ }^{8}$ Para consideraciones adicionales sobre esta posibilidad, ver Pereira (2009). Para una revisión crítica de la ceguera atencional como una forma de amnesia inatencional, ver Wolfe (1999).

${ }^{9}$ Los modelos de comprensión tradicional de la relación entre conciencia y atención normalmente suponen una distinción de tres tipos o niveles de procesamiento que un estímulo o input sensorial puede alcanzar: (i) un nivel inconsciente, (ii) un nivel desatendido y (iii) un nivel atendido que sería el único que se constituye como experiencia consciente. Sin embargo, este modelo ortodoxo parece estar en conflicto directo con evidencia empírica según la cual podemos atender un estímulo de manera inconsciente (Kentridge et al., 2004, 2008). Si esto es así, aún necesitaríamos utilizar la expresión "inconsciente" para caracterizar algunos inputs que no alcanzan a procesarse de manera consciente, pero que sin embargo son atendidos de hecho durante la experiencia. Es por este motivo que Lamme (2003) ha propuesto un nuevo esquema conceptual alternativo más coherente con la evidencia empírica que distingue entre (i) inputs conscientes, (ii) inputs inconscientes y (iii) atención.
} 
alguna respecto de si un estímulo particular alcanza o no a ser experimentado conscientemente. Donde sí tiene incidencia la selección atencional es posibilitando un reporte consciente de estímulos y determinando "si los ítems son guardados de una forma lo suficientemente estable (memoria de trabajo) como para permitir posteriormente un reporte o una comparación con una escena subsecuente" (Lamme, 2003, p. 13).

Si la propuesta anterior es correcta, entonces los casos de ceguera inatencional no deberían interpretarse como fenómenos asociados a la ausencia de conciencia durante una experiencia, sino como una deficiencia en un dominio o capacidad muy específica: la memoria de trabajo (working memory). En efecto, la vinculación entre atención y memoria de trabajo no es nueva y es aceptada incluso por aquellos que defienden TNA. Prinz, por ejemplo, afirma que "la atención puede ser identificada con los procesos que permiten que la información se codifique en la memoria de trabajo" (2011, p. 184). Esta estrecha vinculación entre atención y memoria de trabajo parece coherente con la interpretación que Lamme (2003) hace de los casos de ceguera inatencional. Por ejemplo, en el experimento del gorila (Simons \& Chabris 1999) es posible que los observadores sean conscientes de su presencia, pero al no atender este estímulo no registrarían la información de manera estable en la memoria de trabajo. Esto explicaría por qué estos eventos supuestamente conscientes se borran u olvidan rápidamente y por qué un número significativo de observadores no son capaces de reportar dichos estímulos. Podríamos estar en presencia de un caso de ignorancia epistémica por parte de los observadores o simplemente de una forma de consciencia demasiado desestructurada como para ser utilizada cognitivamente en niveles superiores:

"Para explicar la ignorancia del sujeto no solo necesitamos decir que cualquier diferencia fenoménica que el gorila desatendido haga es una diferencia que el sujeto no es capaz de usar respondiendo la pregunta del experimentador acerca de si algo extraño sucedió en la escena. Podría ser que es una diferencia fenoménica que es inutilizable epistémicamente porque se olvida inmediatamente (...) o podría alternativamente tratarse de una diferencia que es inutilizable porque es demasiado desestructurada e inicial como para ser movilizada epistémicamente" (Mole, 2009, p. 38).

Desde un punto de vista teórico, el modelo interpretativo de Lamme (2003) permite formular dos afirmaciones muy importantes. En primer lugar, al parecer muchos estímulos que de hecho procesamos de manera consciente se desvanecen rápidamente y no pueden ser reportados si la atención no los selecciona permitiendo que la memoria de trabajo los codifique. La atención sería una suerte de portero o conserje (Awh et al., 2006) que determina qué información es procesada en el siguiente nivel. En segundo lugar, habría que reconocer la existencia de dos formas de ser consciente de un estímulo o dos modos de conciencia. Una conciencia difícilmente reportable, vulnerable y de corta duración, y una forma de conciencia más estable en la cual la información puede ser reportada fácilmen$\mathrm{te}^{10}$.

\footnotetext{
${ }^{10}$ Esta distinción entre dos formas de conciencia está estrechamente vinculada a la propuesta filosófica de Block (2007), quien distingue a su vez entre una "conciencia fenoménica" y una "conciencia de acceso cognitivo", otorgándole un rol decisivo a los mecanismos de atención. A juicio de Block, "la fenomenología
}

Por otra parte, Lamme (2003) refuerza estas consideraciones de índole más bien teórica con evidencia neurocientífica. La clave estaría en distinguir entre lo que se conoce como el "barrido de alimentación hacia delante", o feedforward sweep (en adelante: FFS), y el procesamiento recurrente de información visual (en adelante: RP). Debido a que los procesos FFS son necesariamente inconscientes, la conciencia visual -ya sea en su nivel fenoménico o de accesibilidad cognitiva - surgiría con la ocurrencia de RP. Sería precisamente en un dominio consciente donde se produce una transición entre una conciencia desestructurada y una de registro epistémico:

"El tipo de información del cual uno es consciente fenoménicamente se sitúa entre el procesamiento de barrido hacia delante (inconsciente) y el procesamiento recurrente global (conciencia de acceso). Como he dicho, la información de la cual uno es consciente fenoménicamente es de corta vida, vulnerable y es una forma de experiencia visual no fácilmente reportable (Lamme, 2003, p. 3). En contraste, la "conciencia de acceso" (esto es, la conciencia que acompaña nuestras experiencias normales) es más estable y reportable con facilidad" (Raftopoulos, 2009, pp. 41-42).

La tesis de Raftopoulos (2009) implica que cuando RP es local y limitado solo cabría hablar de una conciencia fenoménica vulnerable, que ignoramos desde una perspectiva epistémica y que por lo tanto no posibilita reporte verbal alguno. La accesibilidad cognitiva a esta información solo ocurriría una vez que RP se globaliza incorporando áreas nemónicas y de salida. La atención sería precisamente la responsable de la transición entre dos formas de conciencia, ya que ella selecciona y determina el registro de los estímulos en la memoria de trabajo.

\section{Comentario final}

La posibilidad de una forma de conciencia visual no constreñida por la atención es de vital importancia para nuestra discusión. Si la atención efectivamente opera con independencia de los mecanismos que determinan si una experiencia es o no consciente, entonces los estudios en torno a fenómenos como la ceguera inatencional no logran establecer concluyentemente la tesis TNA para la conciencia visual. Efectivamente, parece haber buenas razones para pensar que la ceguera inatencional y otros fenómenos asociados a la percepción visual son consistentes con interpretaciones alternativas que solo sugieren un déficit de atención o de memoria de trabajo, pero en ningún caso la ausencia de conciencia.

\section{Agradecimientos}

Agradezco el apoyo financiero otorgado por el gobierno de Chile, mediante el Proyecto Fondecyt Iniciación 11090001.

ciertamente sobrepasa la accesibilidad" (2007, p. 487) y son precisamente los mecanismos de atención los que permiten el tránsito desde una conciencia fenoménica a una de acceso cognitivo. Para una lectura crítica de la viabilidad de esta distinción, ver Prinz (2011). 


\section{Referencias}

Awh, E., Vogel, E. K. \& Oh, S. H. (2006). Interactions between attention and working memory. Neuroscience, 139, 201-208.

Blackmore, S. J., Brelstaff, G., Nelson, K., Troscianko, T. (1995). Is the richness of our visual world an illusion? Transsaccadic memory for complex scenes. Perception, 24, 1075-1081.

Block, N. (2007). Consciousness, accessibility, and the mesh between psychology and neuroscience. Behavioral and Brain Sciences, 30, 481-499.

Driver, J. \& Mattingly, J. B. (1998). Parietal neglect and visual awareness. Nature Neuroscience, 1, 17-22.

Kentridge, R. W., Nijboer, T. C. W., \& Heywood, C. A. (2008). Attended but unseen: Visual attention is not sufficient for visual awareness. Neuropsychologia, 46, 864-869.

Kentridge, R. W., Heywood, C. A. \& Weiskrantz, L. (2004). Spatial attention speeds discrimination without awareness in blindsight. Neuropsychologia, 42, 831-5.

Mack, A. \& Rock, I. (1998). Inattentional Blindness. MIT Press.

Mole, C., Smithies, D. and Wu, W. (2011). Attention: philosophical and psychological essays. Oxford University Press.

Mole, C. (2010). Attention is cognitive unison. Oxford University Press.

Mole, C. (2009). Attention. The Stanford Encyclopedia of Philosophy (Fall 2009 Edition). Edward N. Zalta (Ed.) Disponible en http://plato.stanford.edu/archives/fall2009/entries/attention/

Lamme, V. A. F. (2003). Why visual attention and awareness are different. Trends in Cognitive Sciences, 7, 12-18.

Lavie, N. (2007). Attention and consciousness. En Max Velmans \& Susan Schneider (Eds.) The Blackwell Companion to Consciousness. Oxford: Blackwell Publishing Ltd.

O’Regan, J. K. \& Noë, A. (2001). A Sensorimotor Approach to Vision and Visual Consciousness. Behavioral and Brain Science, 24, 883-975.

Pereira, F. (2009). Perceptual Content and Unattended Visual Features. Ideas y Valores, Colombia, 140.

Prinz, J. (2011). Is Attention Necessary and Sufficient for Consciousness?. En Christopher Mole, Declan Smithies \& Wayne Wu (Eds.) Attention: Philosophical and Psychological Essays. Oxford: Oxford University Press.

Raftopoulos, A. (2009). Cognition and Perception. Cambridge, MA: MIT Press.

Rensink, R. A., O'Regan, J. K., Clark, J. J. (2000). On the failure to detect changes in scenes across brief interruptions. Visual Cognition, 7: 127-145.

Simons, D. \& Chabris, C. (1999). Gorillas in our midst: Sustained inattentional blindness for dynamic events. Perception, 28, 1059-1074.

Wolfe, J. M. (1999). Inattentional amnesia. En V. Coltheart (Eds.) Fleeting Memories: Cognition of Brief Visual Stimuli. Cambridge, MA: MIT Press. 